\title{
Ophthalmologische Embryologie und Teratologie - einst und jetzt
}

\author{
Ophthalmic Embryology and Teratology - Past and Present
}

eingereicht 29.4.2014 akzeptiert 30.4.2014

Bibliografie

DOI http://dx.doi.org/ 10.1055/s-0034-1368579 Klin Monatsbl Augenheilkd 2014; 231: 718-719 @ Georg Thieme Verlag KG Stuttgart . New York · ISSN 0023-2165

Korrespondenzadresse Prof. Dr. Jens Martin Rohrbach Eberhard-Karls-Universität Tübingen

Department für Augenheilkunde, Forschungsbereich Geschichte der Augenheilkunde/Ophthalmopathologisches Labor

Schleichstraße 6-12

72076 Tübingen

Tel.: +49/(0)7071/2984761

Fax: $+49 /(0) 7071 / 294762$

Martin.Rohrbach@

med.uni-tuebingen.de

\section{License terms}

Die nachfolgenden Untersuchungen der Bonner Kolleginnen befassen sich mit der regelmäßigen Untersuchung fetaler Augen [1]. Weltweit dürfte es nur sehr wenige Institutionen geben, die sich ebenfalls in dieser „Routine“ üben. In Deutschland ist die Bonner Universitäts-Augenklinik die einzige. Ist das Forschungsgebiet deshalb „ophthalmologisch irrelevant“? Die Autorinnen beantworten diese Frage hinreichend im Abschnitt „Klinische Relevanz“.

Fehlbildungen des Auges und seiner Adnexe spielen an größeren Augenkliniken immer noch eine nicht unwesentliche Rolle. Die Gestalt erklärt sich, wie schon Goethe meinte, maßgeblich durch die Entwicklung, der klinische Aspekt der Fehlbildung mithin zu einem wesentlichen Teil durch die Embryologie. Das Paradebeispiel hierfür sind sicher die auf dem inkompletten Verschluss der Augenbecherspalte beruhenden Kolobombildungen. Für andere Abweichungen von der normalen Form wie beispielsweise das „Hornhautstaphylom-Vorderkammeragenesie-Mikrophakie-Syndrom“ (HVMS) kann zumindest eine embryologische Hypothese formuliert werden [2].

Obwohl die Zahl der Kinder in Westeuropa abgenommen hat, Infektionen sowie Umweltbelastungen während der Schwangerschaft (wahrscheinlich) zurückgegangen sind und sich unsere Erkenntnisse über die genetischen Grundlagen enorm erweitert haben, besteht der Eindruck, dass okuläre Fehlbildungen in den letzten Dekaden nicht seltener geworden sind. Genauere Zahlen liegen in Ermangelung eines entsprechenden Registers indes nicht vor. Die gegenüber früher häufigere Konsanguinität bei den Eltern als Folge der weltweiten „kulturellen Durchmischung“ und das Konsumverhalten - man denke nur an die Alkoholembryopathie bzw. -fetopathie - mögen in diesem Zusammenhang eine Rolle spielen. Die Ursachenklärung nicht nur der ophthalmologischen Fehlbildungen ist immer noch schwierig; oft bleibt sie ergebnislos. Dennoch sollte sie durch morphologische Untersuchungen versucht werden, wenn sich denn die Gelegenheit dazu bietet. Ophthalmopathologie und Ophthalmoteratologie könnten dabei durchaus zu einem besseren Verständnis des ganzen, mit Augenmanifestation einhergehenden Syndroms beitragen.

Die normale Entwicklung des Auges ist seit bald 100 Jahren phänotypisch weitgehend aufgeklärt. Ihre „Essentials“ wurden 2012 noch einmal von den Regensburger Anatomen Tamm und Ohlmann hervorragend zusammengefasst [3]. Die nachfolgenden embryologischen Untersuchungen haben über die teratologischen Aspekte hi- naus perspektivisch das Potenzial, in Verbindung mit der Humangenetik zur weiteren Aufklärung der ,genetischen Abfolge der physiologischen Augenentwicklung“ beizutragen.

Die Forschung unterlag und unterliegt, wie bereits Albrecht von Graefe (1828-1870) 1865 festgestellte, immer auch Modeerscheinungen [4]. Ophthalmoembryologie und -teratologie waren über Dekaden hinweg „modern“ und sie haben man muss heute wohl eher sagen hatten - in Deutschland eine lange Tradition, die vor allem mit Namen wie Friedrich August von Ammon (1799-1861) [5] (๑ Abb. 1), Wilhelm Manz (1833-1911), Eugen von Hippel (1867-1939) [6], Aurel von Szily (1880-1945) [7], Richard Seefelder (1875-1949) [8], Günther Badtke (19101967) und Manfred Tost verbunden ist. Die großartigen ophthalmoteratologischen Werke von Badtke und Tost [9] sowie von Frederic Jakobiec [10] sind mehr als 25 Jahre alt. Neuere, vergleichbare Werke sind dem Autor nicht bekannt. Ja, es könnte derzeit wohl auch niemand mehr ein derartiges Buch verfassen. Spielte die normale und pathologische Entwicklung des Auges in älteren Lehrbüchern der Ophthalmologie wie z.B. „dem Axenfeld“ in allen seinen Auflagen von 19091992 mit 3-8\% des Buchumfangs noch eine signifikante Rolle [11], so kommen moderne Lehrbücher gänzlich ohne die Entwicklungsgeschichte des Auges aus. Und für die Facharztanerkennung benötigt es in Zeiten von IVOM und refraktiver Chirurgie, wie die „1000 Fragen“ suggerieren [12], offenbar keinerlei Verständnisses der Augenentwicklung mehr. Insoweit bewegen sich Martina Herwig und Karin Löffler mit ihren Untersuchungen, ganz wider den Zeitgeist, auf „ophthalmologisch vergessenem Terrain“. Den Wert der Arbeit schmälert das nicht. Im Gegenteil!

\section{Danksagung}

$\nabla$

Der Autor dankt seinen anatomischen Lehrern Johannes W. Rohen und Elke Lütjen-Drecoll, Erlangen, welche durch inspirierende Vorlesungen und Diskussionen das Interesse an der (ophthalmologischen) Embryologie geweckt haben. An die (zum größeren Teil genannten) Kollegen, die sich des nicht einfachen Gebiets der Entwicklungslehre des Auges in der Vergangenheit angenommen haben, sei dankbar erinnert. 


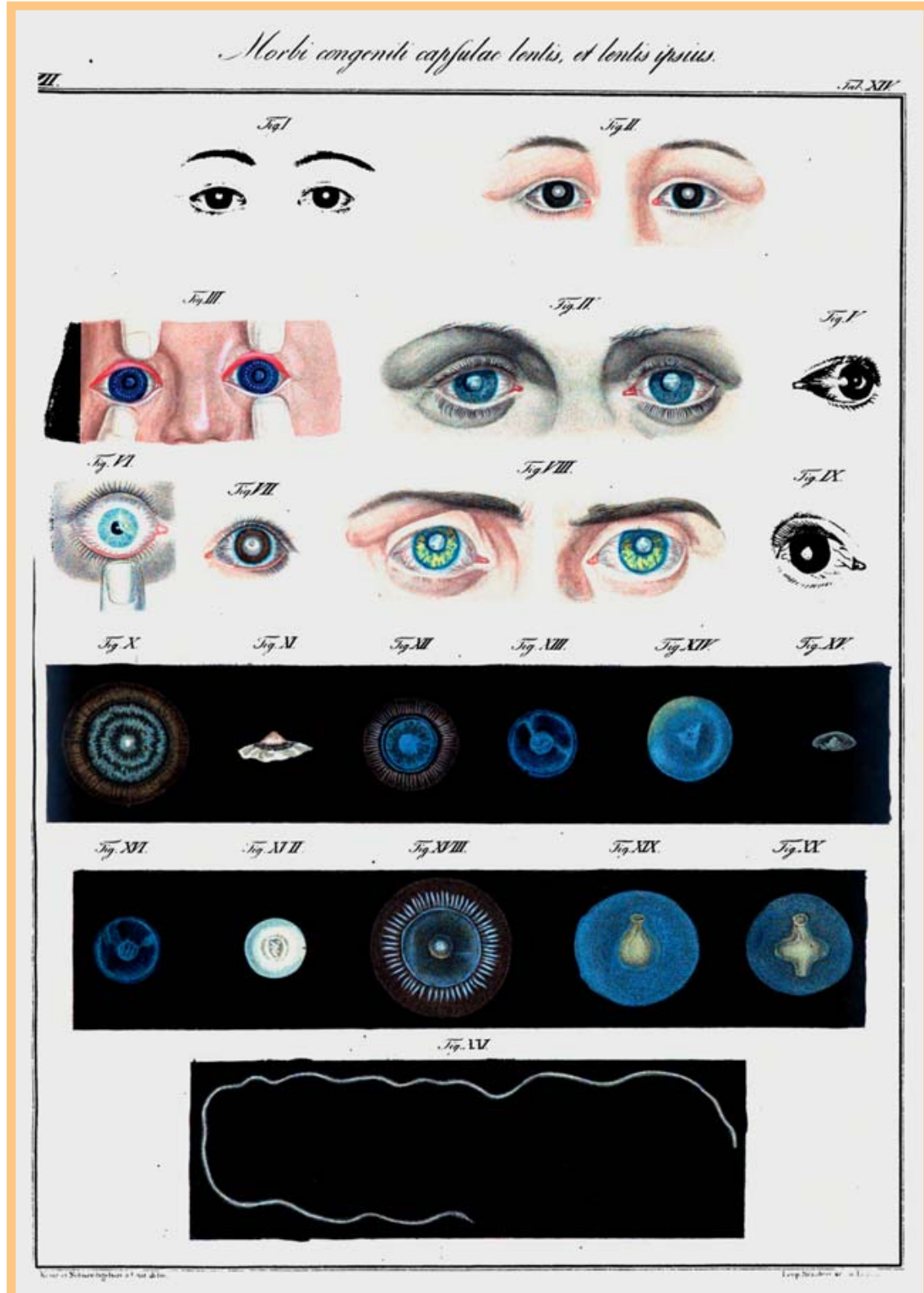

Abb. 1 Friedrich August von Ammon: Kongenitale Erkrankungen der Linsenkapsel und der Linse selbst, 1841. Im untersten Bild ist ein aus einer kongenitalkataraktösen Linse entbundener Wurm dargestellt. (Aus [5].)

\section{Interessenkonflikt \\ $\nabla$}

Nein.

\section{Literatur}

1 Herwig MC, Löffler KU. Routineuntersuchung fetaler Augen - wie und warum? Klin Monatsbl Augenheilkd 2014; 231: 720-728

2 Rohrbach JM, Süsskind D, Szurman P et al. Hornhautstaphylom-Vorderkammeragenesie-Mikrophakie-Syndrom (kongenitales anteriores Staphylom). Klin Monatsbl Augenheilkd 2006; 223: 168-175

3 Tamm ER, Ohlmann A. Entwicklung des menschlichen Auges. Ophthalmologe 2012; 109: 911-925

4 Rohrbach JM. Forschung und Fortschritt in der Augenheilkunde seit 1850 - Versuch einer historisch-kritischen Analyse. Klin Monatsbl Augenheilkd 2013; 230: 1036-1043

5 von Ammon FA. Klinische Darstellungen der Krankheiten und Bildungsfehler des menschlichen Auges, der Augenlider und der Thränenwerkzeuge nach eigenen Beobachtungen und Untersuchungen. Dritter Theil: Klinische Darstellungen der angeborenen Krankheiten des Auges und der Augenlider. Berlin: G. Reimer; 1841
6 von Hippel E. Entwicklungsgeschichte und angeborene Anomalien. In: Axenfeld T, Hrsg. Lehrbuch der Augenheilkunde. Jena: Gustav Fischer; 1909: 203-221 (Des Weiteren auch in der 2. bis 8. Auflage 1910-1935)

7 von Szily A. Modelle zur normalen Entwicklungsgeschichte und Missbildungslehre des Auges. Klin Monatsbl Augenheilkd 1930; 85: 210218

8 Seefelder R. Die Entwicklung des menschlichen Auges/Die Mißbildungen des menschlichen Auges. In: Schieck F, Brückner A, Hrsg. Kurzes Handbuch der Ophthalmologie, Band 1. Berlin: Julius Springer; 1930: 476-630

9 Badtke G, Tost M. Normale Entwicklung des menschlichen Auges/Mißbildungen des menschlichen Auges. In: Velhagen K, Hrsg. Der Augenarzt, Band XI. Leipzig: VEB Georg Thieme; 1986

10 Jakobiec FA. Ocular anatomy, embryology and teratology. Philadelphia: Harper \& Row; 1982

11 Rohrbach JM. Von der Evolution eines ophthalmologischen Lehrbuchs: „Der Axenfeld“ 1909-1992. Klin Monatsbl Augenheilkd 2013; 230: 832-842

12 Kampik A, Grehn F, Messmer EM. Facharztprüfung Augenheilkunde: 1000 kommentierte Prüfungsfragen. Stuttgart: Thieme; 2014 